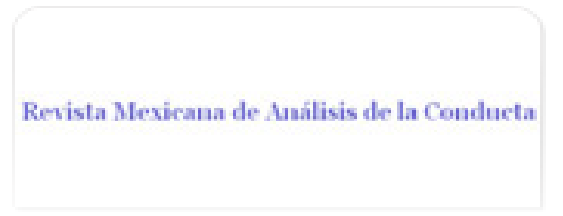

Revista Mexicana de Análisis de la Conducta ISSN: 0185-4534

editora@rmac-mx.org

Sociedad Mexicana de Análisis de la Conducta México

Cuevas Aguirre, Eduardo; Hernández-Pozo, María del Rocío

Evaluación de la impulsividad en fumadores: una revisión sistemática

Revista Mexicana de Análisis de la Conducta, vol. 34, núm. 2, diciembre, 2008, pp. 293-311

Sociedad Mexicana de Análisis de la Conducta

Guadalajara, México

Disponible en: http://www.redalyc.org/articulo.oa?id=59311115009

Cómo citar el artículo

- Número completo

- Más información del artículo

- Página de la revista en redalyc.org

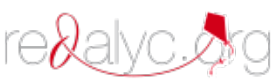

Sistema de Información Científica

Red de Revistas Científicas de América Latina, el Caribe, España y Portugal Proyecto académico sin fines de lucro, desarrollado bajo la iniciativa de acceso abierto 


\title{
EVALUACIÓN DE LA IMPULSIVIDAD EN FUMADORES: UNA REVISIÓN SISTEMÁTICA ${ }^{1}$
}

\author{
IMPULSIVITY ASSESSMENT IN SMOKERS: A SYSTEMATIC REVIEW \\ EDUARDO CUEVAS AGUIRRE \\ Y MARÍA DEL ROCÍO HERNÁNDEZ-POZO \\ PROGRAMA DE DOCTORADO EN PSICOLOGÍA, \\ UNAM-IZTACALA, MÉXICO
}

\begin{abstract}
RESUMEN
El estudio del concepto de impulsividad, por su complejidad, ha sido de interés para la Economía, Sociología, Psicología y Medicina. Existen diferentes instrumentos de medición, que podemos clasificar en los que son de tipo auto-reporte y los que emplean paradigmas conductuales. Los estudios sobre la impulsividad y el tabaquismo son relativamente nuevos y pueden ser útiles para entender la relación entre esa tendencia y el comportamiento tabáquico. Es posible que la impulsividad sea un factor que impida la cesación del consumo de tabaco y que esa tendencia contribuya a las recaídas. La presente revisión sistemática tiene como objetivo principal, la recopilación de las diferentes formas en que se ha evaluado la impulsividad en la población fumadora. A partir de la búsqueda en las bases PUBMED, SCOPUS, SCIENCEDIRECT, SPRINGER y ERIC se encontró un total de 3025 artículos relacionados con la impulsividad de los cuales 700 la evaluaban directamente. Solo 106 relacionaban la impulsividad con la conducta de fumar y de estos solo 20 evaluaron la impulsividad directamente en población fumadora. Se concluye que la utilización conjunta de pruebas de ejecución conductual y
\end{abstract}

1. Proyecto de Investigación en Aprendizaje Humano, UNAM-Iztacala, México. Este artículo fue el producto de un seminario de investigación supervisada que el primer autor cursó bajo la dirección de la segunda autora, sobre el tema de su proyecto de investigación doctoral. Para comunicarse con los autores escribir a: herpoz@servidor.unam.mx y edycuevas@yahoo.com.mx

Recibido: 16 de Septiembre, 2008. Revisado: 17 de Octubre, 2008. Aceptado: 10 de Diciembre, 2008. 
auto-reporte para la medición de la impulsividad, permite una mejor comprensión y explicación del fenómeno de interés.

Palabras clave: impulsividad, conducta impulsiva, medidas de laboratorio, auto-reporte, medidas conductuales, fumadores

\begin{abstract}
Due to its complexity, the study of the concept of impulsivity has been of interest for Economy, Sociology, Psychology and Medicine. Different measures are used to conduct research in impulsivity, these techniques are either selfreports or behavioral paradigms. Studies dealing with impulsivity and tobacco consumption are relatively new and they could be useful to understand the relationship between this tendency and smoking. It is possible that impulsivity is a factor that prevent cessation of tobacco consumption and that this tendency contributes for relapses. The present systematic review has as its central goal to discuss in detail different forms used in the literature to assess impulsivity among smokers. Based on the search on data bases such as PUBMED, SCOPUS, SCIENCEDIRECT, SPRINGER and ERIC, 3025 articles were found related with impulsivity, from which 700 directly evaluated it. Only 106 articles dealt with impulsivity related to smoking behavior and among them only 20 provided measures focused directly on impulsivity among smokers. The review concludes that the joint use of behavioral performance task plus self-reports for assessment of impulsivity, allows a better grasp in the comprehension and explanation of the target phenomena.
\end{abstract}

Key words: impulsivity, behavioral-impulsivity, laboratory measures, selfreport, behavioral measures, smokers.

\title{
ANTECEDENTES
}

La Organización Mundial de la Salud (OMS), en el caso del consumo de tabaco señala que hay casi 1,100 millones de fumadores en el mundo. Del total de la población mundial, $30 \%$ de los adultos son fumadores y de éstos, $4 \mathrm{mi}$ llones de personas fallecen al año, lo que equivale a la muerte de casi 11,000 personas diarias (OMS/OPS, 2006). En México, se registran 165 muertes cada día a causa del tabaquismo y numerosos casos de enfermedades asociadas, las cuales podrían prevenirse, por lo que se han desarrollado diferentes estrategias para solucionar dicho problema de salud, como son: programas relacionados con los impuestos, programas publicitarios, programas educativos dirigidos a la población en general y programas de tratamiento 
para ayudar a los fumadores a dejar esta adicción (Kuri, 2002; Kuri-Morales, González-Roldan, Hoy \& Cortés Ramírez, 2006; Rubio, 2001).

De acuerdo a la Encuesta Nacional de Adicciones (2002), más de 16 millones de personas $(23.5 \%)$ son fumadores, $17.4 \%$ ex fumadores y más de la mitad $(59.1 \%)$ manifestaron ser no fumadores. Se estima que de los individuos en tratamiento, el $75-80 \%$ presentan alguna recaída en los seis primeros meses (Carmody, 1992). Así mismo, de los pacientes que intentan dejar de fumar, el $30 \%$ fallan en el primer mes de tratamiento y cerca del $80 \%$ de los pacientes que logran abstenerse, muestra alguna recaída en el primer año (Guisa, Díaz, \& Souza-Machorro, 2004; Hunt \& Bespalec, 1974).

Diversos estudios se han dirigido a conocer los factores de riesgo que conducen a la recaída de los fumadores. En dichos estudios se ha encontrado que factores como el estrés, las relaciones sociales con otros fumadores, el consumo de alcohol o café, la presencia de emociones negativas, así como la depresión, ansiedad e impulsividad dificultan lograr la abstinencia y ocasionan la recaída (Brown, Legues, Kahler \& Strong, 2002; Doran, Spring, McChargue, Pergadia \& Richmond, 2004; Krall, Garvey \& Garcia, 2002; Shiffman, 1982; Zvolensky, Bonn-Miller, Bernstein \& Marshall, 2006).

El estudio del concepto de impulsividad, por su complejidad, ha sido de interés para diferentes disciplinas como son la Economía, Sociología, Psicología y Medicina (Arce \& Santisteban, 2006). Éste constructo refiere a diferentes matices según el contexto diagnóstico y teórico al que se adscribe, matices que deben ser tenidos en cuenta, tanto para la utilización adecuada de los distintos instrumentos de medida, como en las intervenciones terapéuticas que corresponden a diferentes concepciones teóricas (Forcada, Pardo \& Bondía, 2006).

Moller, Barrat, Dougherty, Schmitz y Swann (2001), definen la impulsividad como la incapacidad para considerar las consecuencias a largo plazo y el decremento de la sensibilidad para las consecuencias negativas, con reacciones rápidas e imprevistas hacia los estímulos antes de completar el procesamiento de la información.

De esta forma podemos considerar a la impulsividad como la elección entre alternativas de respuesta concurrentemente disponibles, que producen inmediatez pero con un menor reforzamiento, es decir, el sujeto elige una opción que produce reforzadores pequeños inmediatos en lugar de los que producen reforzadores grandes pero demorados. Mientras que su inverso es el autocontrol, el cual es definido como la elección de la respuesta que produce una alta ganancia a un largo plazo (Neef, Bicard \& Endo, 2001).

Para la evaluación de la impulsividad en diversos trastornos, como los relacionados con sustancias, parafilias, trastornos antisocial y límite de la personalidad, hiperactividad, trastorno disocial, esquizofrenia, manía, trastorno explosivo intermitente, cleptomanía, piromanía, juego patológico, trico- 
tilomanía y trastorno del control de los impulsos no especificado del DSM-IV; se han desarrollado varios instrumentos de medición, que de acuerdo a su aproximación podemos clasificarlos en los de tipo auto-reporte y en los conductuales (ver tabla 1).

Entre los cuestionarios de auto-reporte más utilizados se encuentran seis, a saber:

El Cuestionario de impulsividad 17 (Eysenck, Pearson, Easting \& Allsopp, 1985), el cual consiste en 54 reactivos distribuidos en tres subescalas: Impulsividad (19 ítemes), aventura (16 ítemes) y empatia (19 ítemes). La puntuación obtenida es la suma de los ítemes para cada uno de los factores, cuenta con una confiabilidad aceptable; su alfa de Crobach es de 0.83 (Leshem \& Glicksohn, 2007).

La Escala de impulsividad de Barratt (BIS-11, Patton, Stanford \& Barratt, 1995), consta de 30 reactivos divididos en tres factores que miden: 1) la inatención e inestabilidad cognitiva, 2) impulsividad motora y carencia de perseverancia y 3) carencia de autocontrol e intolerancia de complejidad cognitiva. Cuenta con un alfa de Crobach de 0.79 (Leshem \& Glicksohn, 2007). A diferencia de la versión 10, la cual constaba de 34 reactivos que se responden con una escala Licker de cuatro opciones (nunca, ocasionalmente, a veces, siempre, cada opcion con un valor numérico correspondiente a 0,1,2,3,4 donde 4 indica la respuesta más impulsiva). La suma total de las respuestas muestra el nivel de impulsividad para cada una de las tres subescalas: impulsividad motora (se refiere a una tendencia a actuar sin pensar), impulsividad cognitiva (se relaciona con una propensión a tomar decisiones rápidamente) e impulsividad no planificada (se identifica con una tendencia a no trazar planes y realizar tareas de forma descuidada) (Barratt, 1985).

El Cuestionario Multidimensional de la Personalidad (MPQ por sus sigla en inglés, Patrick, Curtín \& Tellegen, 2002), el cual es un cuestionario comprensivo de la personalidad, que cuenta con tres mega-factores (emocionalidad positiva, emocionalidad negativa y compulsión) y ocho factores primarios, de los cuales solo el apartado de reactivos sobre compulsión se consideran como impulsividad.

La Escala de Conducta Impulsiva UPPS (Whiteside \& Lynam, 2001), se compone de 45 reactivos que evalúan cuatro factores de la impulsividad (urgencia, carencia de premeditación, carencia de perseverancia y sensación de búsqueda).

El Cuestionario de Personalidad Tridimensional (CPT, Cloninger, 1986), que es un cuestionario de 100 reactivos dicotómicos de falso-verdadero, evaluando tres dimensiones de la personalidad como son la búsqueda de novedad, evitación del daño y dependencia de recompensa, las cuales son componentes de la impulsividad. 
El Inventario de Temperamento y Carácter (ITC-125), es un cuestionario autoaplicado y consta de 125 ítem a los que se responde verdadero o falso, cuantifica cuatro dimensiones del temperamento que comprenden: capacidad para evitar el daño, búsqueda de novedad, dependencia de la recompensación y persistencia, y tres dimensiones del carácter, las cuales abarcan: autodeterminación, disposición a la cooperación y auto-trascendencia (Cloninger, 1987).

La Escala de personalidad Karolinska (EPK, Weinryb 1992), que comprende 135 preguntas agrupadas en 15 escalas: ansiedad somática, ansiedad psíquica, tensión muscular, psicastenia, inhibición de la agresión, evitación de la monotonía, impulsividad, socialización, desapego, deseabilidad social, agresión verbal, agresión indirecta, irritabilidad, sospecha y culpa (Schalling \& Edman, 1993).

Tabla 1

Tipos de pruebas utilizadas para la evaluación de la impulsividad.

\begin{tabular}{ll}
\hline \multicolumn{1}{c}{ Pruebas de auto-reporte } & \multicolumn{1}{c}{ Pruebas conductuales } \\
\hline 1. Cuestionario de impulsividad I-7 & 1. Tarea de Parar a la señal, TPS \\
2. Escala de impulsividad de Barratt BIS- & 2. Tarea de Ir no-ir, TINI \\
II & 3. Tarea de Ejecución continua de \\
3. Cuestionario multidimensional de la & Conners TEC \\
personalidad CMP & 4. Tarea de Demora-Descuento TDD \\
4. Escala de conducta impulsiva UPPS & 5. Tarea Experimental de Descuento \\
5. Cuestionario de Personalidad & TED \\
Tridimensional CPT & 6. Tarea Simple de Impulsividad TSI \\
6. Escala de personalidad Karolinska & 7. Tarea de Globo Análogo a Riesgo \\
EPK & TGAR \\
& 8. Paradigma de Stroop PS \\
\hline
\end{tabular}

Con respecto a las evaluaciones conductuales, podemos encontrar dos tipos de paradigmas: el de inhibición de estímulos y el de demora del reforzador o descuento. Entre las primeras ubicamos a tres procedimientos: la tarea de irno ir, la prueba de ejecución continua de Conners y el paradigma de Stroop.

La Tarea de ir no-ir es una prueba en la cual los sujetos aprenden a dar una respuesta ante la presencia de una señal de "siga", sin embargo, en una segunda serie de ensayos, se presenta la indicación de no-seguir mientras que en otra serie de ensayos se presenta una señal de "alto" simultáneamente presentado con la señal de seguir o antes que esta. La Tarea de parar a la señal es idéntica a la de ir no-ir, solo con la diferencia de que la señal de alto es presentada después de la señal de siga y entre más cerca esta la señal de alto del momento de responder, el participante debe inhibir la respuesta (Winstanley \& Robbins, 2006). 
La Prueba de Ejecución Continua de Conners (PEC) consiste en una tarea en la cual se presentan 500 letras sucesivas durante 400 mseg con un intervalo entre estímulo de $600 \mathrm{mseg}$, teniendo un tiempo total de 8 minutos para la tarea. El sujeto debe responder cada vez que aparece una $X$ precedida por una A. Eso ocurre en 50 ocasiones, pero en otras 50 veces aparece la letra $X$ no precedida por $A$ y otras 50 ocasiones las $A$ no seguidas de $X$. Las medidas de impulsividad son los errores de omisión (número de AX no detectadas) y los de comisión (número de veces que se responde en ausencia de una X precedida por una A) (Félix, 2006).

La prueba Stroop, consiste en un error o retraso provocado al nombrar un determinado color cuando éste se presenta al sujeto como el color en que esta escrita una palabra, que a su vez nombra a otro color, en este caso incongruente con el de la tinta en que esta exhibida dicha palabra. Consta de tres partes que se realizan separadamente durante 45 segundos cada una. La primera condición de palabras consiste en leer las palabras rojo, verde y azul escritas en negro sobre fondo blanco. La segunda condición de colores consiste en nombrar el color de Xs que puede ser rojo, verde o azul. En la tercera condición de color-palabras aparecen las palabras rojo, verde y azul escritas en colores diferentes. La variable que mide impulsividad es la interferencia, que se calcula restando el número de respuestas en la condición de color-palabras del de la condición colores (Félix, 2006).

En resumen, este tipo de pruebas se caracterizan por la presencia de un grupo de opciones ante las cuales la persona tiene que responder de acuerdo a la presencia de una señal y detenerse ante la presencia de otro estímulo determinado, es decir, poder inhibir la respuesta ante una señal.

En el segundo grupo de pruebas conductuales de impulsividad se encuentran pruebas en las cuales los participantes deben elegir entre una recompensa pequeña inmediata, o bien, una recompensa mayor pero demorada. La diferencia entre este grupo de pruebas radica en la forma de presentarlas a los participantes (Reynolds, Ortengren, Richards \& Wit, 2006; Winstanley, Eagle \& Robbins, 2006) e incluyen la tarea de demora descuento, la tarea experimental de descuento, la tarea simple de impulsividad y la tarea del globo análogo a riesgo.

La Tarea de Demora-Descuento (TDD), es una prueba en la cual se presentan diferentes ensayos en los cuales se tiene una opción de seleccionar un valor relativo de un reforzador inmediato contra uno demorado, es decir, los participantes pueden elegir el obtener una cantidad de dinero grande, después de un tiempo (demora), o bien una cantidad pequeña, pero inmediata (Reynolds, Ortengren, Richards \& Wit, 2006).

La Tarea Experimental de Descuento (TED), es una prueba conductual que evalúa la demora de descuento en tiempo real, a través de cuatro bloques de elecciones de retraso (por ejemplo: $0,7,15$ y 30 s), en los cuales los 
participantes escogen entre una recompensa pequeña e inmediata, o bien una recompensa grande, pero demorada. Durante la tarea los participantes eligen entre obtener puntos o cuenta monetaria que se consideran opciones equivalentes (Krishnan-Sarin et al., 2007).

La Tarea Simple de Impulsividad (TSI), es un modelo de laboratorio en donde se presentan a los sujetos una serie de ensayos (por ejemplo, 50), y en cada ensayo los participantes tienen que elegir entre un reforzador pequeño inmediato o uno grande y demorado (Reynolds, Ortengren, Richards \& Wit, 2006).

La Tarea del Globo Análogo a Riesgo (TGAR), es una prueba de toma de riesgo, en el cual los participantes pueden ganar o perder puntos cambiables por dinero. Se presenta en la pantalla de una computadora un globo el cual hay que inflar apretando un botón, el tamaño del globo es el mismo de la ganancia, solo que a cierto número de presiones el globo se rompe y por consiguiente se pierden los puntos, los participantes pueden transferir su dinero al banco al terminar el ensayo y antes de continuar con el siguiente ensayo con lo cual pueden ir acumulando sus ganancias (Reynolds, Ortengren, Richards \& Wit, 2006).

Adicionalmente, los estudios sobre elección han identificado algunos procesos específicos relacionados con el autocontrol. Uno de ellos es el descuento temporal o descuento de la demora (DD) en el cual se evidencia una tendencia a preferir recompensas pequeñas pero inmediatas, en contraste con consecuencias grandes pero más demoradas, la cual ha sido un modelo de elección del autocontrol Rachlin \& Green, 1972)

Ahora bien, los hallazgos de diversas investigaciones muestran la relación entre impulsividad y diversos trastornos. En el caso de la adicción a drogas, no es clara del todo la evidencia sobre si esta relación se debe a los efectos específicos del abuso de sustancias o a la frecuente comorbilidad con trastornos de la personalidad como el límite y el antisocial según criterios del DSM-IV.

Se han encontrado niveles elevados de impulsividad en las personas que intentan suicidarse, los adolescentes con problemas alimentarios, personas con conducta agresiva, jugadores compulsivos y pacientes con desórdenes severos de personalidad y con déficit de atención (Dawe, Gullo \& Loxton 2004; Dom, De-Wilde, Hulstijn \& Sabbe, 2007). Recientemente, se ha vinculado la investigación del tema de impulsividad con el consumo de drogas, identificando la conducta impulsiva como factor de alto riesgo para el tabaquismo, alcoholismo y la drogadicción (Dawe \& Loxton, 2004).

Los estudios que exploran las relaciones entre impulsividad y tabaquismo son relativamente nuevos, pero esos estudios (Bickel, Odum \& Madden, 1999; Doran, Spring, McChargue, Pergadia \& Richmond, 2004; Mitchell, 1999; Skinner, Aubin \& Berlin, 2004) proporcionan suficiente evidencia de 
que los fumadores tienden a presentar tendencias impulsivas, siendo este factor, el responsable de impedir la cesación del consumo de tabaco y de aumentar la probabilidad de la recaída, ya que se ha documentado que los fumadores con un nivel elevado de impulsividad recaen tempranamente o bien, fracasan en el proceso de cesación del tabaquismo.

La presente revisión sistemática tiene como objetivo principal, la recopilación de las diferentes formas en que se ha evaluado la impulsividad en la población fumadora, con la finalidad de presentar los diferentes instrumentos que se han empleado en la literatura científica para valorar la impulsividad y si esta tendencia difiere en las personas fumadoras en comparación con otro tipo de población. Por lo cual se integran cuantitativamente los resultados de los estudios que incluyen estas dos variables de interés y de esta manera contar con un panorama de las pruebas utilizadas con mayor frecuencia y su alcance en el estudio de la impulsividad, se consideró este constructo y no el de autocontrol, debido a que se buscó la medición tanto de la personalidad impulsiva como la manifestación conductual de ésta. Aunque existe una variedad de estudios en donde se relacionan el consumo de sustancias y la impulsividad, son pocos los referentes a tabaco y que por el tipo de droga (legal y de fácil acceso) resulta ser un potente reforzador difícil de igualar, además de que el estudio de éstas variables es de importancia, ya que en la actualidad los tratamientos para la cesación del tabaquismo presentan una alta recaída $(80 \%)$ dentro del primer año.

\section{MÉTODO}

Búsqueda de la literatura

El proceso de la búsqueda de los estudios relacionados con la evaluación de la impulsividad se obtuvo consultando las siguientes fuentes: PUBMED, SCOPUS, SCIENCEDIRECT, SPRINGER y ERIC, en el período comprendido de 1988 a 2008, de las cuales se seleccionaron las investigaciones mediante la utilización de los siguientes descriptivos: impulsivity, behavioral-impulsivity, laboratory measures, self-reported, behavioral measures apareados con el término smoking (también se buscaron en su equivalente en español).

De los estudios encontrados se incluyeron en esta revisión los que cumplían los siguientes criterios: 1) ser estudios comparativos entre grupos, 2) contar con datos cuantitativos, 3) utilizar algún tipo de evaluación conductual o de auto-reporte para la impulsividad y 4) utilizar a población fumadora sin otro tipo de padecimiento físico y/o mental. El proceso de búsqueda permitió encontrar un total de 20 estudios que cumplieron con los criterios establecidos publicados en el periodo señalado. 
Codificación de los estudios.

Los estudios fueron codificados de acuerdo a las características del tipo de prueba empleada para la valoración de la impulsividad, considerando el tipo de diseño, tamaño de la muestra y los resultados relevantes.

\section{RESULTADOS}

La búsqueda de artículos referentes al tema de impulsividad en las bases de datos mencionadas, generó un total de 3025 investigaciones, de las cuales $700(23 \%)$ evaluaron la impulsividad con algún tipo de prueba (auto-reporte o conductual) y $106(3.5 \%)$ se relacionaron con la conducta de fumar. De estos últimos, el $18 \%$ (20) evaluaron la impulsividad en población fumadora, del cual el $40 \%$ (ocho) fueron de tipo conductual, el $45 \%$ (nueve) fueron de autoreporte y un $15 \%$ (tres) utilizaron ambos tipos de pruebas.

De los diferentes instrumentos utilizados para la valoración de la impulsividad en la población fumadora mediante el auto-reporte, la encontrada con mayor frecuencia fue la Escala de Impulsividad de Barratt (BIS11), mientras que en la de tipo conductual, fue la Tarea de Descuento-Demora. Estas pruebas han demostrado tener un alto índice de confiabilidad para la medición de la impulsividad, e incluso con otro tipo de poblaciones. Los hallazgos y características de los diferentes estudios analizados se encuentran resumidos en la tabla 2. 


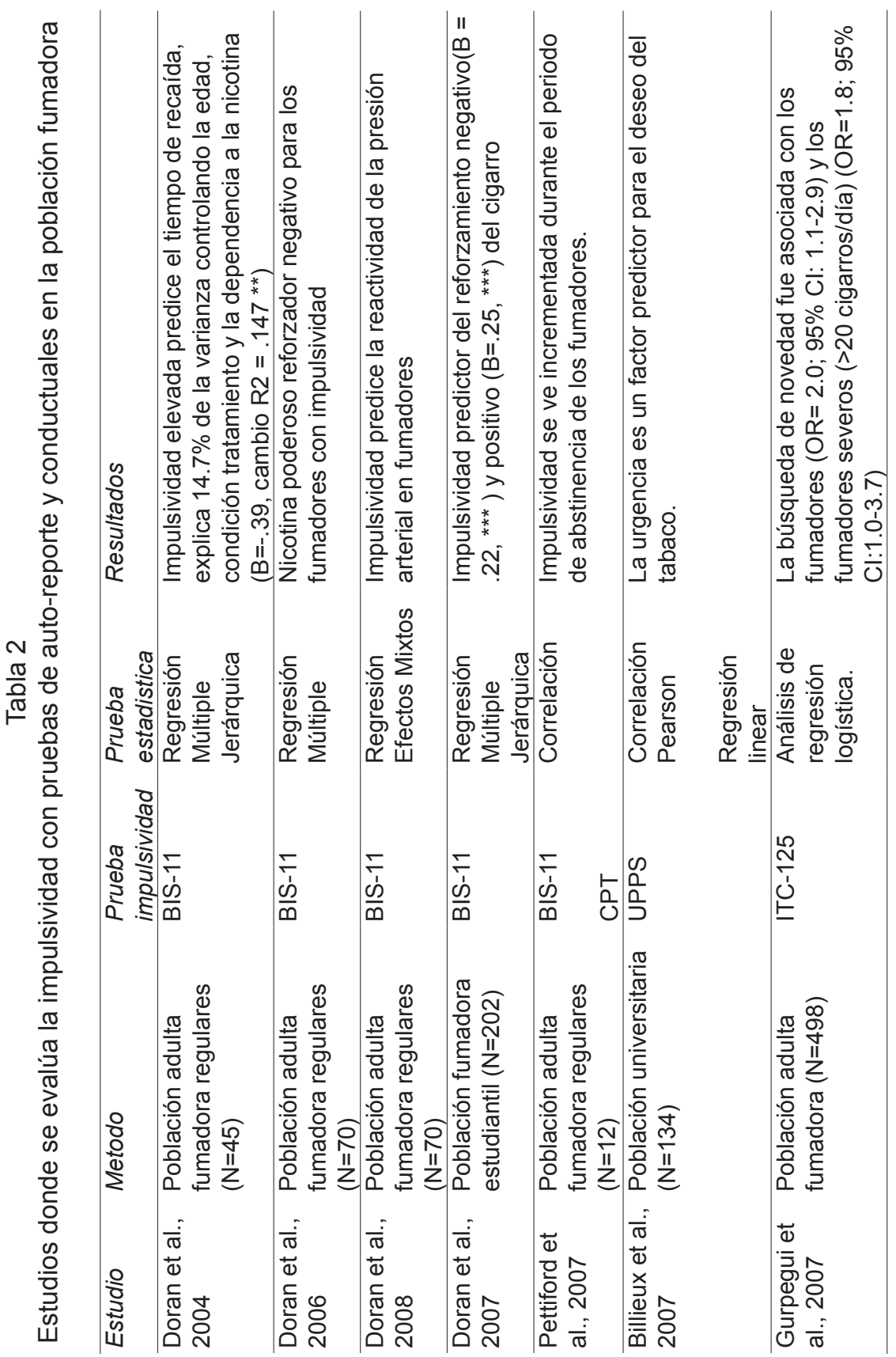



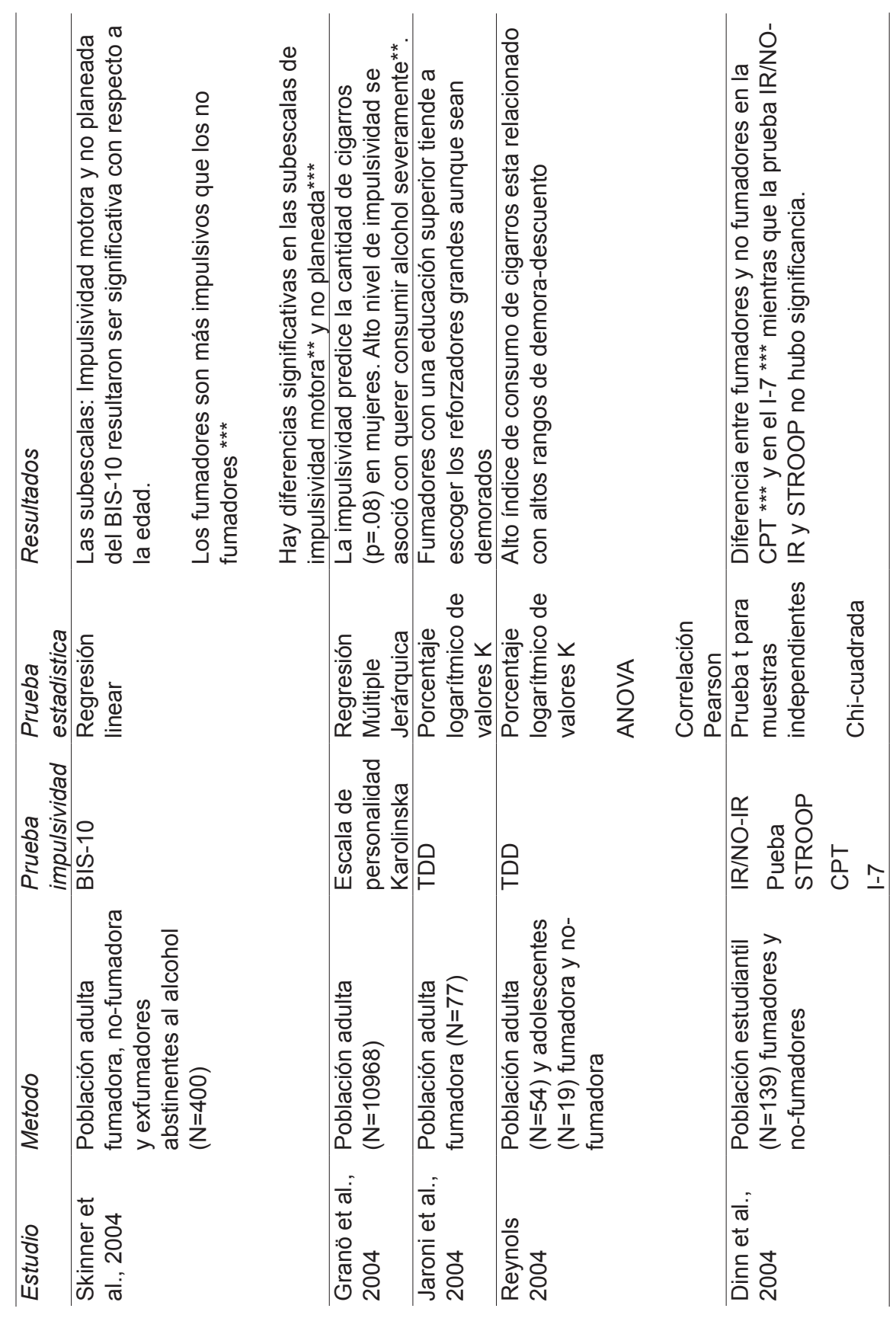

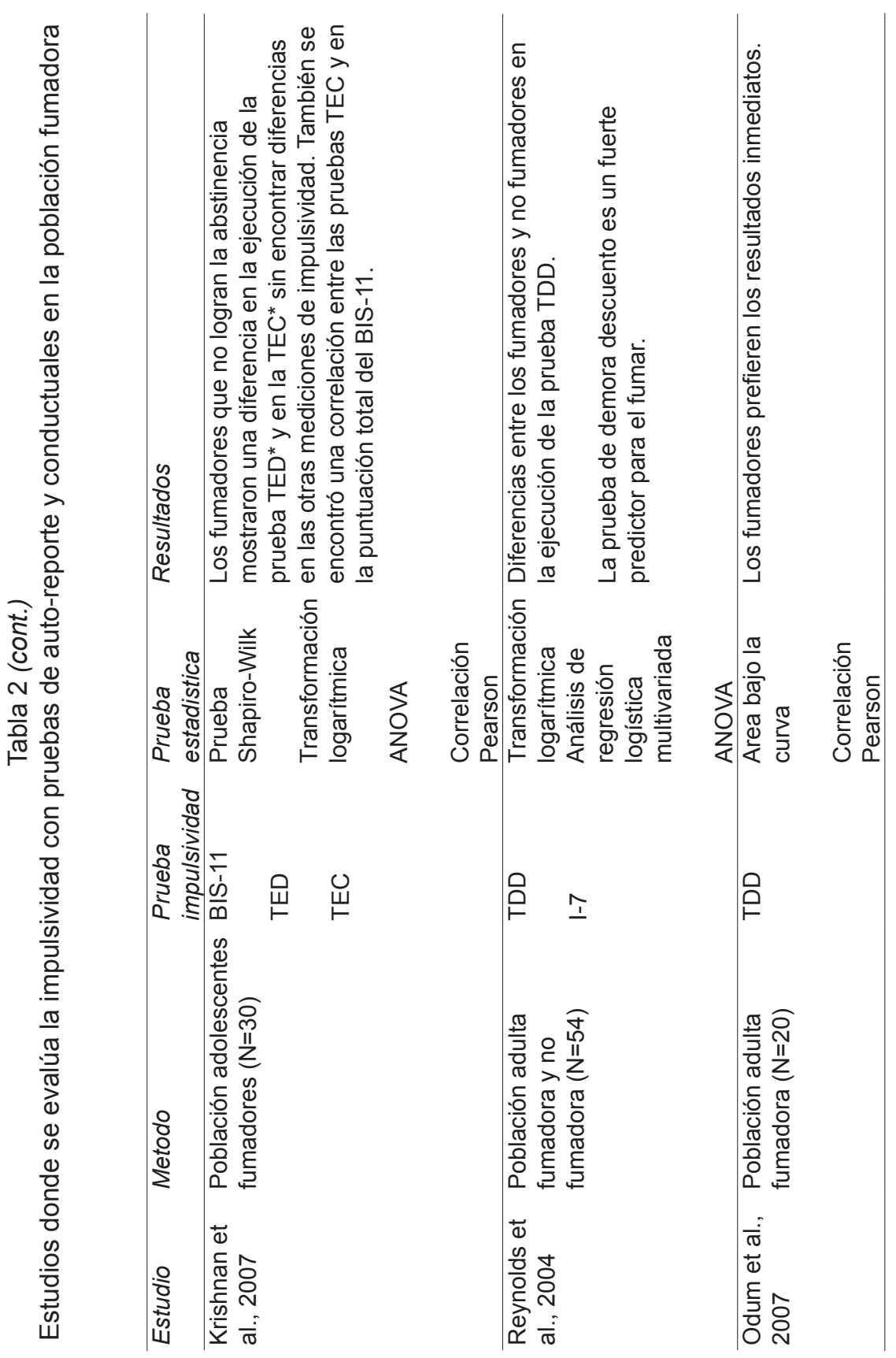


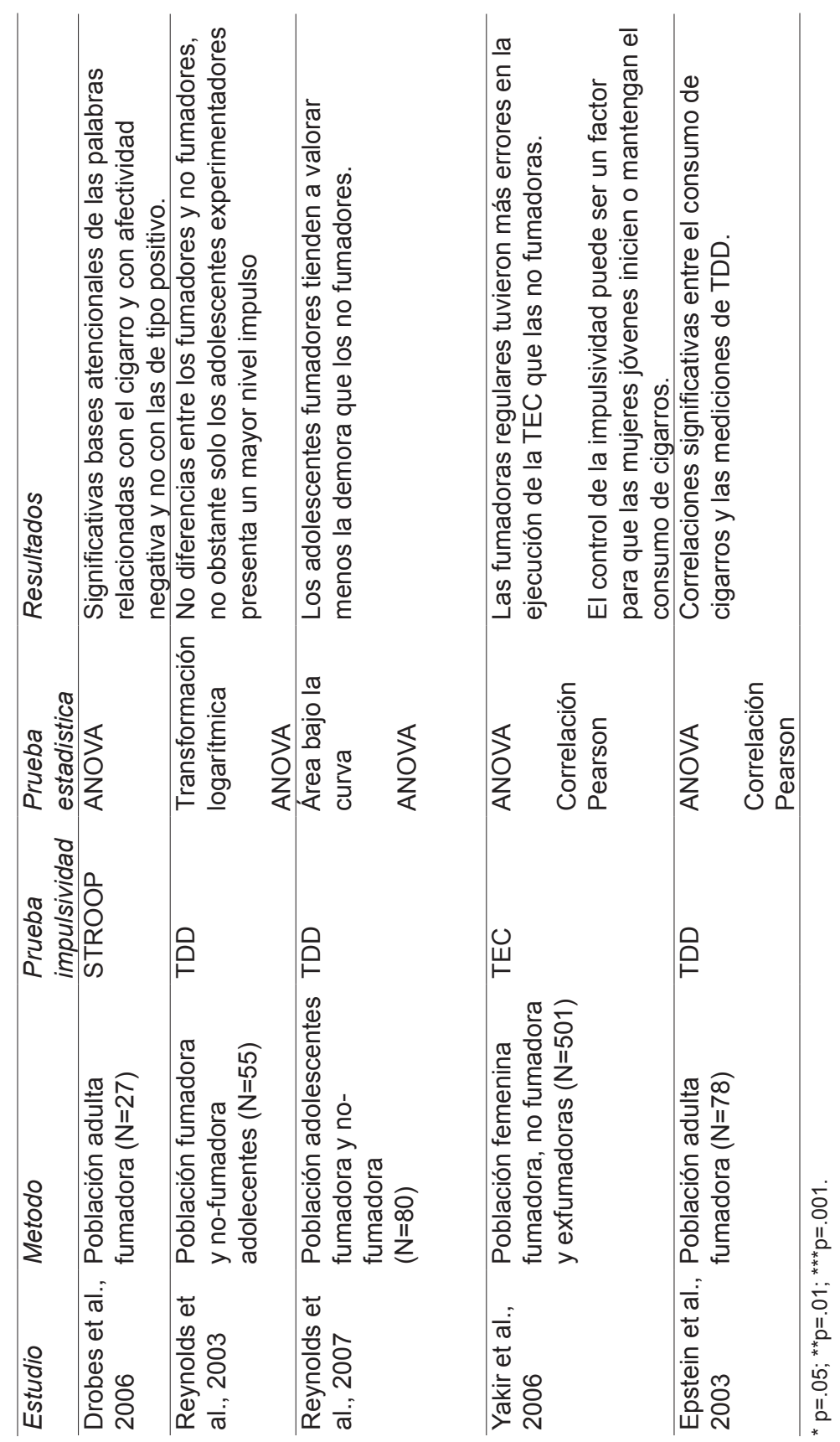


La mayoría de los estudios incluidos en la tabla 2, fueron diseños de grupo, encontrándose diferencias significativas entre los fumadores y los no fumadores. De igual forma se pudo observar que existe una relación entre el inicio y/o mantenimiento de la conducta de fumar y los niveles de impulsividad. También, se encontró una relación directa entre el nivel de impulsividad y el consumo de cigarros y el tiempo que transcurre antes de la recaída, es decir a mayor nivel de impulsividad mayor consumo y menor tiempo de abstinencia (Doran et al., 2004; Granö et al., 2004; Skinner et al., 2004; Doran et al., 2007; Krishnan-Sarin et al., 2007).

Son pocos los estudios que utilizan conjuntamente los dos tipos de pruebas (auto-reporte y conductual) para la valoración de la impulsividad, en esta revisión sólo fueron tres. En el caso de las mediciones conductuales de la impulsividad se ha encontrado que los fumadores tienden a escoger los reforzadores inmediatos aunque sean pequeños en lugar de los demorados, por lo que consideramos que la aplicación de una prueba conductual aunada al auto-reporte, proporciona información más completa ya que no solo nos informa de la tendencia-autocontrol-impulsividad ya definida, sino que además permite el registro de tendencias de inatención y de impulsividad motora. De esa forma el empleo simultáneo de pruebas verbales y conductuales de este tipo, es una aproximación preferible a evaluar impulsividad con las pruebas verbales y conductuales por separado (Reynolds 2004; Odum et al., 2007; Reynolds et al., 2006; Reynolds et al., 2007)

Se encontraron otro tipo de pruebas de personalidad en las cuales existe una subescala de impulsividad, sin embargo no mostraron diferencias significativas entre los fumadores y no fumadores (Granö et al., 2004; Billieux et al., 2007).

Por último se encontraron artículos en los cuales se median la impulsividad y el consumo de cigarros, pero se descartaron de esta revisión, debido a que la población presentaba además un diagnóstico psiquiátrico. Cabe mencionar que las pruebas de auto-reporte como son el: I-7, CMP, CPT, EPK y ITC-125 resultan tener mayor validez cuando se pretende evaluar el perfil de personalidad y no sólo un aspecto de ésta.

\section{CONCLUSIONES}

En la revisión realizada se encontraron varios artículos que evaluaban la impulsividad de una forma conductual o bien con pruebas de auto-reporte, pero para este trabajo fueron incorporados solo los estudios que evaluaban la impulsividad en la población fumadora, dando un total de 20 estudios, los cuales presentan evidencia de que el tema de la impulsividad en el inicio, mantenimiento y cesación del tabaquismo es de reciente interés, así como una correlación directa entre la impulsividad y el consumo de cigarros, de- 
mostrando la existencia de diferencias significativas entre las personas fumadoras y los no fumadores en ambos tipos de pruebas (Dinn et al., 2004; Reynolds et al., 2004. Skinner et al., 2004).

Es importante señalar que el estudio de la impulsividad en varias patologías, es de reciente interés, aunque es complejo su estudio, la aplicación de paradigmas conductuales en su evaluación han permitido entender mejor el fenómeno y fortalecer las mediciones hechas mediante el auto-reporte, ya que en éstas a pesar de contar con validez y confiabilidad, existe el error que puede presentar los sujetos al contestarlas, debido a sesgos probables en la población objeto, a diferencia de los paradigmas conductuales, en los cuales la ejecución motora permite el control de la predisposición por parte de los sujetos hacia el tipo de evaluación sobre sus tendencias conductuales.

Entre las pruebas de auto-reporte la que se utiliza con mayor frecuencia es la Escala de Impulsividad de Barratt, ya que es la que ha demostrado mayor confiabilidad y validez, utilizada en trastornos psiquiátricos y otro tipo de padecimientos en donde la impulsividad juega un papel importante. Aunque existen otras pruebas que incluyen a la impulsividad como una subescala, estas han demostrado tener mayores desventajas que la de Barratt o bien son específicas para un sólo tipo de población, por lo que su generalidad es limitada.

Por otra parte, las tareas con el paradigma de demora-descuento han demostrado ser confiables para la evaluación de la impulsividad, además de contar con la ventaja de poder ser aplicadas en cualquier tipo de población con o sin características especiales. Entre las pruebas conductuales la que se encontró con mayor frecuencia fue la tarea de demora-descuento (Richards, Zhang, Mitchell \& Wit, 1999).

La utilización de ambas pruebas de auto-reporte y de ejecución conductual para la medición de la impulsividad, permite un mejor conocimiento y explicación del fenómeno, como lo demuestran los estudios en los que se aplicaron ambos tipos de prueba (Dinn et al., 2004; Reynolds et al., 2004; Krishnan-Sarin et al., 2007).

En resumen, se puede concluir que el estudio de la impulsividad es de importancia para la explicación de los trastornos relacionados con el consumo de sustancias, ya que juega un papel primordial en el proceso de inicio, mantenimiento, cesación y recaída de la adicción, la cual debe ser evaluada desde un enfoque integral tanto de la personalidad como de la conducta impulsiva, por lo que la utilización de pruebas que cubran esta demanda como son la Escala de Impulsividad de Barratt (BIS11) y la Tarea de Descuento-Demora (TDD), resultan una herramienta eficaz para el entendimiento y tratamiento del fenómeno. 


\section{REFERENCIAS}

American Psychiatric Association. (1994). Diagnostic and statistical manual of mental disorders: DSM-IV. 4th ed. Washington: American Psychiatric

Association.

Arce, E., \& Santisteban, C. (2006). Impulsivity: A review. Psicothema, 18(2), 213-220.

Barratt, E. S. (1985). Impulsiveness substrait: Arousal and information processing. In J. T. Spence y C. E. Izard (Eds.), Motivation, emotion, and personality. (pp. 137146). Amsterdam: International Union of Psychological Science.

Bickel, W. K., Odum, A. L., \& Madden, G. J. (1999). Impulsivity and cigarette smoking: Delay discounting in current, never, and ex smokers. Psychopharmacology, 146, 447-454.

Billieux J., Van der Linden M., \& Ceschi G. (2007). Which dimensions of impulsivity are related to cigarette craving? Addictive Behaviors, 32, 1189-1199.

Brown, R. A. Legues, C. W., Kahler, C. W., \& Strong D. R. (2002). Distress tolerance and duration of past smoking cessation attempts. Journal of Abnormal Psychology, 111, 180-185.

Carmody, T. P. (1992). Preventing relapse in the treatment of nicotina addiction: Current sigues and future directions. Journal of Psychoactive Drugs, 24,131-158.

Cloninger, C. R. (1986). A unified biosocial theory of personality and its role in the development of anxiety status. Psychiatric developments, 4(3), 167-226.

Cloninger, C. R. (1987). A systematic method for clinical description and classification of personality variants. A proposal. Archives General Psychiatry, 44, 573-588.

Dawe, S., \& Loxton, N. (2004). The role of impulsivity in the development of substance use and eating disorders. Neuroscience and Biobehavioral Reviews, 28, 343-351.

Dawe, S., Gullo, M., \& Loxton N. (2004). Reward drive and rash impulsiveness as dimensions of impulsivity: Implications for substance misuse. Addictive Behaviors, 29, 1389-1405.

Dinn, W., Aycicegi, A., \& Harris, C. (2004). Cigarette smoking in a student simple: Neurocognitive and clinical correlates. Addictive Behaviors, 29, 107-126.

Dom, G., De-Wilde, B., Hulstijn, W., \& Sabbe, B., (2007). Dimensions of impulsivity behavior in abstinent alcoholics. Personality and Individual differences, 42(3), 465-476.

Doran, N., Spring, B., McChargue, D., Pergadia, M., \& Richmond, M. (2004). Impulsivity and smoking relapse. Nicotine and Tobacco Research, 6(4), 641-647.

Doran, N., McChargue, D., Spring, B., Vander V., Werth. J., \& Richmond, M. (2006). Effect of nicotine on negative affect among more. Experimental and Clinical Psychopharmacology, 14(3), 287-295.

Doran, N., McChargue, D., \& Spring, B. (2008). Effect of impulsivity on cardiovascular and subjective reactivity to smoking cues. Addictive Behaviors, 33, 167-172.

Doran, N., McChargue, D., \& Cohen L. (2007). Impulsivity and the reinforcing value of cigarette smoking. Addictive Behaviors, 32, 90-98.

Drobes, D., Elibero, A., \& Evans D. (2006). Attentional bias for smoking and affective stimuli: A Stroop Task Study. Psychology of addictive behaviors, 20(4), 490-495. 
Encuesta Nacional de Adicciones de México, (2002). Secretaria de Salud, Dirección General de Epidemiología, Instituto Mexicano de Psiquiatría "Ramón de la Fuente".

Epstein, L. H., Richards, J. B., Saad, F. G., Paluch, R. A., Roemmich, J. N., \& Lerman, C. (2003). Comparison between two measures of delay discounting in smokers. Experimental and Clinical Psychopharmachology, 11(2), 131-138.

Eysenck, S. B. G., Pearson, P. R., Easting G., \& Allsopp, J. F. (1985). Age norms for impulsiveness, venturesomeness and empathy in adults. Personality and Individual Differences, 6, 613-619.

Félix, M. V. (2006). Recursos para el diagnóstico psicopedagógico del TDAH y comorbilidades. Revista Electrónica de Investigación Psicoeducativa, 10(4), 623-642.

Forcada, C. R., Pardo, P. N. \& Bondía, S. B. (2006). Impulsividad en dependientes de cocaína que abandonan el consumo. Adicciones, 18(2), 111-118.

Granö, N., Virtanen, M., Batear, J., Elovainio, M., \& Kivimäki, M. (2004). Impulsivity as a predictor of smoking and alcohol consumption. Personality and Individual Differences, 37, 1693-1700.

Guisa, V. M., Díaz, B., Salgado, L., \& Souza-Machorro, M. (2004). Terapéutica antiadictiva: utilidad de sus factores de predicción. Revista Mexicana Neurociencias, 5(6): 581-598.

Gurpegui, M., Jurado D., Luna, J., Fernández-Molina, C., Moreno-Abril, O., \& Gálvez R. (2007). Personality traits associated with caffeine and smoking. Progress in neuro-psychopharmacology \& Biological psychiatry, 31, 997-1005.

Hunt, W. A., \& Bespalec, D. A. (1974). An evaluation of current methods of modifying smoking behavior. Journal Clinic of Psychology, 431-438.

Jaroni, J., Wright, S., Lerman, C., \& Epstein, L. (2004). Relationship between education and delay discounting in smokers. Addictive Behaviors, 29, 1171-1175.

Krishnan-Sarin S., Reynolds B., Duhig A. M., Smith A., Liss T., McFetridge A., CavaIlo D. A., Carroll K. M., \& Potenza M. N. (2007). Behavioral impulsivity predicts treatment outcome in a smoking cessation program for adolescent smokers. Drug Alcohol Depend, 88(1), 79-82.

Krall, E. A., Garvey, A. J., \& Garcia, R. I. (2002). Smoking relapse after 2 years of abstinence:findings from the VA normative aging study. Nicotine and Tobacco Research, 4, 95-100.

Kuri, M. P. (2002). Tabaquismo en México. En Secretaria de Salud (Eds.), Observatorio Mexicano en tabaco alcohol y otras drogas (pp.27-29). México: Consejo Nacional contra las Adiciones.

Kuri-Morales, P., González-Roldan, J., Hoy, M., \& Cortés-Ramírez, M. (2006). Epidemiología del Tabaquismo en México. Salud Pública de México, 48(3), S91-S98

Leshem, R., \& Glicksohn J. (2007). The construct of impulsivity revisited. Personality and Differences, 43,687-691.

Mitchell, S. H. (1999). Measures of impulsivity in cigarette smokers and non-smokers. Psychopharmacology, 146, 455-464.

Moller, F. G., Barrat, E. S., Dougherty, D. M., Schmitz, J. M., \& Swann, A. C. (2001). Psychiatric aspects of impulsivity. American Journal of Psychiatry, 158, 17831793. 
Neef, N. A., Bicard, D. F., \& Endo, S. (2001). Assessment of impulsivity and the development of self-control in students with attention deficit hyperactivity disorder. Journal of Applied Behavior Analysis. 34, 397-408.

Odum, A., \& Baumann A. (2007). Cigarette smokers show steeper discounting of both food and cigarettes than money. Drug and alcohol dependence 91, 293-296.

OMS/OPS, (2006). Estrategia de cooperación con el país México. Organización panamericana de la salud oficina regional de la organización mundial de la salud. Noviembre 2005 [Publicación electrónica]. Disponible en: http://www.paho.org/ english/d/csu/CCSMEX05-11.pdf

Patrick, C. J., Curtín J. J., \& Tellegen, A. (2002). Development and validation of a brief form of the multidimensional personality questionnaire. Psychological Assesment, $14,150-163$.

Patton, J. H., Stanford M. S., \& Barratt, E. S. (1995). Factor structure of the Barratt Impulsiveness Scale. Journal of Clinical Psychology, 51, 768-774.

Pettiford, J., Kozink, R. V:, Lutz, A. M., Kollins, S. H., Rose, J. E., \& McClernon, F. J. (2007). Increases in impulsivity following smoking abstinence are related to baseline nicotine intake and boredom susceptibility. Addictive Behaviors 32(10), 2351-2357.

Rachlin, H. \& Green, L. (1972). Commitment, choice and self-control. Journal of the Experimental Analysis of Behavior, 17, 15-22.

Reynolds, B., Ortengren A., Richards, J. B., \& de Wit H., (2006). Dimensions of impulsive behavior: Personality and behavioral measures. Personality and Individual Differences, 40, 305-315.

Reynolds, B., Richards, J. B., Horn, K., \& Karraker K. (2004). Delay discounting and probability discounting as related to cigarette smoking status in adults. Behavioural Processes 65, 35-42.

Reynolds, B., Karraker, K., Horn, K., \& Richards, J. B. (2003). Delay and probability discounting as related to different stages of adolescent smoking and non-smoking. Behavioural Processes 64, 333-344.

Reynolds, B., Patak, M., \& Shroff, P. (2007). Adolescent smokers rate delay rewards as less certain than adolescent nonsmokers. Drug and Alcohol Dependence, 90, 301-303.

Richards, J. B., Zhang, L., Mitchell S., \& de Wit, H. (1999). Delay and probability discounting in a model of impulsive behavior: effect of alcohol. Journal of the Experimental Analysis of Behavior, 71, 121-143.

Rubio, M. H. (2001). Programa de Acción: Adicciones. Tabaquismo. CONADIC-SSA, México.

Schalling, D., \& Edman, G. (1993). The Karolinska scale of Personality (KSP). An inventory for assessing temperament dimensions associated with vulnerability for psychosocial deviance. Manual, Stockholm: The Department of Psychiatry, The Karolinska Institute.

Shiffman.(1982). Relapse following smoking cessation: A situational analysis. Journal Consulting Clinic Psychology, 50, 71-86.

Skinner, M. D., Aubin, H. J., \& Berlin I. (2004). Impulsivity in smoking, nonsmoking, and ex-smoking alcoholics. Addictive Behaviour. 29(5), 973-978). 
Yakir, A., Rigbi, A., Kanyas, K., Pollak, Y., Kahana, G., Karni, O., Eitan, R., Kertzman, S., \& Lerer, B. (2006). Why do young women smoke? III. Attention and impulsivity as neurocognitive predisposing factors. European neuropsychopharmacology, doi:10.1016/j.euroneuro.2006.09.004.

Weinryb, R. M., Gustavsson, J. P., \& Asberg, M. (1992). The concept of alexithymia: An empirical study using psychodynamic ratings and self-reports. Acta Psych. Scand, 85(2), 153-162.

Whiteside, S. P., \& Lynam, D. R. (2001). The five factor model and impulsivity: Using a structural model of personality to understand impulsivity. Personality and Individual Differences, 30, 669-689.

Winstanley, C. A., Eagle, D. M., \& Robbins, T. W. (2006). Behavioral models of impulsivity in relation to ADHD: Translation between clinical and preclinical studies. Clinical Psychology Review 26, 379-395.

Zvolensky, M., Bonn-Miller, M., Bernstein, A., \& Marshall, E. (2006). Anxiety sensitivity and abstinence duration to smoking. Journal of Mental Health, 15(6), 659-670. 